\title{
Application of Geophysical Methods in Tunnel Exploration
}

\author{
Ding Hai-hong ${ }^{1, a}$, Jiang Wei-wei ${ }^{2, b}$ \\ 1. East China Mineral Exploration and Development Bureau for Non-Ferrous, No.102 Shimenkan \\ Community Qinhuai District in Nanjing, 210007, Jiangsu Province, China; \\ 2. Jiangsu Province JianYuan Geologic Engineering and Investigation Co., Ltd., No.20 Compassion \\ Club Road Gulou District in Nanjing, 210029, Jiangsu Province, China; \\ a.60462291@qq.com, b.76125027@qq.com
}

Key words: geophysical prospecting method; tunnel exploration; geological characteristics Abstract: Tunnel construction is a livelihood project, but the nature of all kinds of complex geological problems will cause some impact on them. Therefore, it is necessary to carry out the geological investigation in order to ensure safety and stability of the tunnel, and the geophysical prospecting method is one of the effective methods of the tunnel survey. This paper introduces several common geophysical prospecting methods in tunnel exploration, analyzes the advantage and disadvantage of various methods, and points out that the survey should not be confined to a certain way, but should be more comprehensive.

\section{Introduction}

In recent years, due to increased demand for infrastructure, road construction by leaps and bounds, the natural moat change into thoroughfare truly. In order to meet the requirements of design, the route in the mountain heavy hill area inevitably requires the use of tunnel engineering, the tunnel under construction meets a variety of complex geological problems. These problems must be found out. And in tunnel exploration methods, geophysical methods are important.

\section{Problems to solve in tunnel survey}

If a area intend to built tunnel, it needs to do geological and engineering survey to find out geological structure background firstly. Then the builders can know whether exists adverse factors, such as seam, ore body, broken width and the hydrological geological features. So tunnel designing can avoid some bad geological phenomenon. Even the tunnel put into use, some special constructions are still in the long process of slow development, posing a potential threat to tunnel engineering. Therefore, if want tunnel engineering to be a beneficial long-term projects, we must monitor of tunnel project in a long time, find hidden problems at any time, and take reasonable measures, to make risks to the minimum.

\section{Common geophysical methods in tunnel survey}

Tunnel survey differs from the single characteristic of mineral exploration, it needs to make detailed layer for earth surface and to explore some specific target objectives, which are likely to be caves, aquifers, or may be faults and cracks. Therefore, there are many geophysical prospecting methods can be used in tunnel survey, the most common methods including seismic exploration ${ }^{[1-5]}$ and high-density resistivity method ${ }^{[1-6]}$, controllable source audio frequency magneto telluric sounding method ${ }^{[4-6]}$, the concentration of radon measurement ${ }^{[7]}$, the ground penetrating radar 
method $^{[8]}$, and so on.

\section{Seismic refraction wave method}

The premise of using seismic exploration method is the differences in elastic properties of rock, strata and the target. The principle is artificial seismic wave excitation (explosives), wave propagation in the underground inhomogeneous medium, when the medium elastic properties of interfaces difference is bigger, the reflection and refraction phenomena appeared, on the ground, feedback of seismic waves are pre placement of seismograph accept analysis and processing, to different interface properties are interpreted, to infer the formation of structural forms.

Seismic refraction wave method buried in a certain arrangement and the certain number of detectors in the ground surface. Detector accepts signal described in the preceding paragraph refraction wave and feedback to the ground signal. Then, the signal is transmitted to the seismic instrument, and the seismic instrument amplify the received wave signal, and the waveform of the refraction wave is stored in the disk. Later through the series pretreatment we can map out the time distance curve. The attenuation of seismic waves in the process of investigation of the refraction wave method can be calculated by the result of refraction data reconciliation. The method can calculate the longitudinal wave velocity, and combined with the geological situation of integrated geophysical interpretation, geological geophysical profile interpretation.

Advantage: Seismic refraction wave method to detect depth is a shallow layer detection category, the method mainly through the wave velocity anomaly analysis to accurate and quantitative investigation of the thickness of the cover layer and the thickness of the loose layer. The strata in the distribution direction of the tunnel axis are stratified, and the classification of the surrounding rock of the tunnel is divided. And by differences in different medium velocity interface proved such as tectonic fracture zone like concealed structure position and the method in shallow buried tunnel investigation has obvious advantages.

Inadequate: Exploration depth is shallow, cannot be applied in deep buried tunnel investigation; unable to track the occurrence of faults; the use of explosives artificial seismic wave excitation, and explosive substances have certain restrictions on the management and construction difficulty increased year by year.

\section{Seismic reflection wave method}

The difference in elastic properties between formation and object is also the premise of seismic reflection wave method, and the seismic source mode is the same as the method of refraction wave, Similar to the reflection wave method to the detector and seismometer installation method and refraction wave method. The difference is, reflected wave detector to collect is seismic wave back from the different formation interface reflection seismic reflection records, the seismograph of amplification. Reflection wave data for processing through the interpretation, filtering, speed analysis and dynamic correction and by spectral analysis to extract velocity data, combined with geological conditions to carry out the comprehensive geological and geophysical interpretation.

Advantage: detection depth is larger than the refraction wave method, applicable in deep buried depth of tunnel exploration, can also be used for detection of overburden and weathered layer thickness and hierarchical classification of surrounding rock, find out the fault, fracture zone, karst and soil cave development.In addition, the fault occurrence can be determined, and the distribution of the structure and the relatively small size of the secondary structure has a certain effect. 
Inadequate: can't directly display the speed data, need to use a series of pretreatment and then analyze the velocity spectrum to extract the velocity data; used as the source of explosives management strictly, the difficulty of obtaining increased year by year.

\section{High density resistivity method}

The electrical differences between underground geological target and the surrounding rock is the premise of high density resistivity method to carry out. The method using artificial method to establish a stable dc field underground, and using established device and booking arrangement scanning observation. By collecting the conduction current distribution in the ground, apparent resistivity analysis to detect the target body occurrence space changes. When measuring the one-time all electrode layout is good, can automatically run and collect data, and to incorporate profile and sounding at an organic whole. Measurement data transmission directly into the computer, can be processing, and interpretation of the measured apparent resistivity profile, which geoelectric sections of various kinds of diagrams are obtained.

Advantage: Working mode diversification, can use several kinds of electrode arrangement, can collect more abundant of information, so as to get more detailed structure information; Function of data preprocessing, profile shapes to visual display, abstract data for visualization; A survey completed the $2 \mathrm{~d}$ data acquisition, at low cost, realize the high efficiency work, obtain the result of rich information, will greatly enhance capacity for exploration. Practical applications, suitable for deep buried deep in the depth of the tunnel detection, especially suitable for tunnel survey, using the apparent resistivity difference relative thickness of the overburden layer and weathered layer and hierarchical classification of surrounding rock, to realize qualitative distinction, and utilization of low apparent resistivity anomaly find out fault, fracture zone, the karst soil caves development situation, in addition, can also be used for the deep water condition analysis.

Inadequate; High density resistivity prospecting are appears as inverted trapezoidal shape, the exploration depth, the greater exploration area is smaller, resulting in prospecting area on both sides there have some blind spots. The restriction of the terrain conditions and testing instruments, the mountains tunnel exploration difficulty is big, and the exploration depth has certain restrictions. Interface can intuitively reflect the objective body shape, but in terms of surface depth from all walks of life situation is still belongs to the qualitative analysis, especially in the complex terrain region, unable to accurately determine the buried depth.

\section{Controlled source audio-frequency magneto telluric sounding (CSAMT)}

The electrical differences between the geological target and surrounding rock is also the premise of CSAMT method to carry out. CSAMT using artificial source, the grounding conductor arranged along a certain direction and will be an audio harmonic current into the underground, after line arranged along the direction of wire distribution and observed along the line perpendicular to each other in the electric field and magnetic field components, and then calculate the apparent resistivity . CSAMT method uses different frequency current to achieve the purpose of detecting different deep.

Advantage: High efficiency; strong signal and strong anti-interference ability, little affected by terrain; Wide exploration depth, generally can reach $1 \sim 2 \mathrm{~km}$; sensitive to low resistivity anomalies with high lateral resolution and strong apparent resistivity resolution. Applications, is suitable for the deep tunnel survey, especially suitable for tunnel hole survey, can grasp the macro grew up deep buried tunnel hole hierarchical situation, fractures, karst and property characteristics. 
Inadequate: The near field effect of CSAMT method is one of the largest factor influence the process of data collection. This effect should be to take remedial measures to reduce the impact, such as the large distance method. Applications, show on the shallow information interpretation accuracy is poorer, abnormal enough refinement, local how solution; Hierarchical aspects also belong to qualitative analysis.

\section{Measurement of radon concentration}

Predominantly uranium isotope radioactive elements contained in the strata, the uranium decay series will happen, eventually forming radon. Local layer in the presence of fracture, fracture, fracture zone, the general will be compared with high concentrations of radon anomaly of surrounding rock, radon measurement is on the basis of concentration difference and to carry out the survey work, so close to the fault fracture zone in the formation.

Radon concentration measurement data processing is simple, the collected data into the computer, the use of drawing software diagram or plan, combined with geological and drilling data, and can map integrated geological and geophysical prospecting.

Advantage: Without placing electrodes, line, etc., portable instrument, simple operation, easy to understand the data quantity.

Inadequate: Application of this method should be on the premise of existence fault fracture zone; Shallow exploration depth.

\section{Geological radar detection method}

Geological radar detection method based on the difference of target body and surrounding rock wave impedance, the specific principle is that different media have different reflection principle in the face of electromagnetic wave, and then doing imaging processing in detection area .

Advantage: It can detect the geology and structures within a certain range in front of the geological exploration, such as lithology, strata in the hole, the distribution of water. It can be used in the hole survey and as a way to supplement seismic electrical magnetic method to provide the basis for subsequent tunnel remediation.

Inadequate: the advance detection distance is not long and the detection depth is shallow.

\section{Development prospect of tunnel survey}

It is not difficult to see the advantage and disadvantage of each kind of geophysical exploration methods. There is no method that can solve all the problems. In the practical application, we also research in the form of integrated geophysical prospecting method, For examples:

(1) Example of survey before the tunnel construction [7]: In Guangzhou Wuzhou Expressway Tunnel survey, Zhou Xiaoyu and other scholars have adopted the method of seismic refraction and reflection wave method. They searched interface information about covering layer and bedrock , and outlined the fault location and attitude. By the method of high density resistivity, it is inferred that there is a general location of karst or fault structure in the presence of high and low resistivity anomalies; then the CSAMT method and the soil radon concentration measurement method were used to confirm the development zone of several fracture zones and joints, and to describe the occurrence and location of the fracture zone. Fracture zone of refraction wave method reflects the characteristics of low velocity, high density resistivity and CSAMT method performance for low apparent resistivity measured radon method shows high radon concentrations, geological geomorphological characteristics for deep valley. The survey results are well consistent with the 
drilling verification, which provides a basis for the construction of the tunnel.

(2)Survey examples after the completion of the tunnel ${ }^{[8]}$ : The newly built railway tunnel karst Loushao zhangjiewan remediation survey, Qing Zhi and other researchers take the surface and tunnel survey and parallel method, the application of magneto telluric sounding method on the ground survey, to survey the surface to tunnel part of karst features; In the tunnel, the use of geological radar exploration technology, aimed at determining the development of karst cave and the exact location of the tunnel relationship. The practice and magneto telluric sounding method reflects the cave part is relatively low resistivity, the method not only from the macro level identified geological features and karst and the groundwater in the vicinity of the main flow channel showed, but karst abnormal tunnel and the relative position between the expression is not very clear. And geological radar reflecting diffraction form strong reflection signals section for cave, geological radar detection results to reflect the former cave anomaly information gives a detailed statement, show anomalies in surrounding rock of the tunnel karst spatial position, both the combination of whole and local, macro and micro, final survey results provide a basis for the subsequent remediation work, remediation results tested show is effective.

To sum up, in practical tunnel exploration application, geological condition is complex and changeful, all kinds of factors influence and link to each other. Therefore, in the selection of geophysical exploration method is more to learn from each other, according to local conditions, complementary advantage. The development trend of tunnel survey should be based on rational analysis, which will be used in many ways.

\section{References}

[1]Huang Pan, Wang Chuan-lei, Liu Bin et al., Application of Comprehensive Geophysical Prospecting Method in the Exploration of High-speed Railway Tunnel [J]. Chinese Journal of Engineering Geophysics, 2009, 6(4): 503 507.

[2]Yang Yong. Application of the Comprehensive Geophysical Exploration on the Tunnel Survey of a Freeway in Fujian Province [J]. Geology of Fujian,2010, 29(2): 128 134.

[3]Zhou Zhu-sheng, Feng Yun. The Comprehensive Geophysical Survey In tunnel Exploration [J]. Progress in Geophysics, 2011, 26(2): 724 731.

[4]Shang Xiao-wei. Application of Geophysical Prospecting Method on the Exploration of Tunnel [J]. West-china Exploration Engineering, 2012, (5): 177 179.

[5]Yang Ke. Analysis on Geophysical Prospection and Drilling Prospection Combination Application in Tunnel Exploration[J]. Shanxi Architecture, 2015, 41(20): 85 87.

[6]Qin Tu-guan, FENG Yi. The Application of 2 Geophysical Exploration Methods to GUANYIYAN Tunnel Construction [J]. Yunnan Geology, 2015, 34(3): 444 448.

[7]Zhou Xiao-yu. Application of Synthetic Geophysical Method in Exploration of Tunnel [J]. Highway Traffic in Guangdong, 2006, (97): 5 9.

[8]Qing Zhi. The Application of Integrated Geophysical Exploration Method of the Karst Defect

Treatment in Lou-Shao Zhangjiawan Railway Tunnel [J]. Resources Environment and Engineering, 2014, 28(2): 193 196. 